\title{
Áreas protegidas no Brasil e no mundo: quadro geral de sua implementação
}

\author{
Protected areas in Brazil and in the world: general picture of its implementation \\ Áreas protegidas en Brasil y en el mundo: marco general de su aplicación
}

Recebido: 28/08/2021 | Revisado: 04/09/2021 | Aceito: 16/09/2021 | Publicado: 17/09/2021

\author{
Augusto César Trigueiro Félix \\ ORCID: https://orcid.org/0000-0001-8223-780X \\ Universidade Federal de Campina Grande, Brasil \\ E-mail: eng.augusto91@gmail.com \\ Isabel Lausanne Fontgalland \\ ORCID: https://orcid.org/0000-0002-0087-2840 \\ Universidade Federal de Campina Grande, Brasil \\ E-mail: isabelfontgalland@gmail.com
}

\begin{abstract}
Resumo
As áreas protegidas são um importante instrumento de conservação dos sistemas ambientais. Tendo em vista a necessidade de divulgar as áreas protegidas enquanto meio de conservação da natureza e consequente manutenção da vida humana, o artigo em questão procurou contribuir para o aprofundamento desta temática, tendo em vista a melhoria do quadro atual na proteção ambiental. Com isso, o artigo tem como objetivo apresentar uma revisão bibliográfica dos principais trabalhos publicados sobre o tema das áreas protegidas tanto em um contexto internacional quanto em um contexto nacional. Percebe-se que a partir da década de 60/70 a quantidade de áreas criadas por ano começa a aumentar. Algumas explicações são possíveis para esse aumento, uma delas se dar pela influência da realização das grandes conferências das nações unidas realizadas neste período. Através das referências trazidas no trabalho, observa-se que mesmo tendo sido indentificado um aumento na criação das áreas, a proteção ambiental ainda carece de atenção dos governantes em vários Estados-Nação.
\end{abstract}

Palavras-chave: Áreas verdes; Legislação ambiental; Conservação ambiental.

\begin{abstract}
Protected areas are an important instrument for the conservation of environmental systems. In view of the need to disseminate protected areas as a means of nature conservation and consequent maintenance of human life, the article in question sought to contribute to the deepening of this theme, with a view to improving the current framework in environmental protection. Thus, the article aims to present a bibliographic review of the main works published on the theme of protected areas both in an international and a national context. It is noticeable that from the 60's and 70's onwards the number of areas created per year started to increase. Some explanations are possible for this increase, one of them being the influence of the great Conferences of the United Nations held during this period. Through the references brought in the work, it is observed that even though an increase in the creation of areas has been identified, environmental protection still lacks the attention of governments in many nations.
\end{abstract}

Keywords: Green areas; Environmental legislation; Environmental conservation.

\section{Resumen}

Las áreas protegidas son un instrumento importante para la conservación de los sistemas ambientales. Dada la necesidad de difundir las áreas protegidas como medio de conservación de la naturaleza y consiguiente mantenimiento de la vida humana, el artículo en cuestión pretendía contribuir a la profundización de este tema, con el fin de mejorar el marco actual en la protección del medio ambiente. Así, el artículo pretende presentar una revisión bibliográfica de los principales trabajos publicados sobre el tema de las áreas protegidas tanto en el contexto internacional como en el nacional. Se observa que a partir de los años 60 y 70 empezó a aumentar el número de zonas creadas por año. Hay algunas explicaciones posibles para este aumento, siendo una de ellas la influencia de las grandes Conferencias de las Naciones Unidas celebradas durante este periodo. A través de las referencias traídas en el trabajo, se observa que aún habiéndose identificado un aumento en la creación de áreas, la protección del medio ambiente aún carece de la atención de los gobiernos en varias naciones.

Palabras clave: Áreas verdes; Legislación ambiental; Conservación ambiental.

\section{Introdução}

As áreas protegidas são um importante instrumento de conservação dos sistemas ambientais. Elas são usadas com esta finalidade desde o século XIX, mas só no século XX é que ficaram populares, muito devido ao aumento da influência de 
organizações internacionais, como a IUCN (International Union for Conservation of Nature), nas políticas dos Estados-Nação. Tal organização até os dias de hoje se apresenta como uma das principais propagadoras da proteção ambiental. No Brasil, as áreas protegidas se popularizaram a partir da criação, no ano 2000, do SNUC (Sistema Nacional de Unidades de Conservação), chegando a um crescimento exponencial na criação dessas áreas nos anos posteriores. Nesse sistema existem dois grandes grupos de unidades de conservação (UC), as áreas de Proteção Integral e as de Uso Sustentável. A diferença principal desses grupos se dar no grau de exploração dos recursos permitido no interior dessas áreas, no primeiro é permitido certo nível de utilização de seus recursos e no segundo não é permitido utilização.

Tendo em vista a necessidade de divulgar as áreas protegidas enquanto meio de conservação da natureza e consequente manutenção da vida humana, o artigo em questão procurou contribuir para o aprofundamento desta temática, tendo em vista a melhoria do quadro atual na proteção ambiental. Com isso, o artigo tem como objetivo apresentar uma revisão bibliográfica dos principais trabalhos publicados sobre o tema das áreas protegidas tanto em um contexto internacional quanto em um contexto nacional. Para atingir tal objetivo foi mostrado o quadro institucional das áreas protegidas no Brasil e das organizações internacionais que incentivam esta política pelo mundo, assim como, utilizou-se o banco de dados fornecido pela IUCN, que contém dados das áreas protegidas em várias partes do mundo, e o banco de dados fornecido pelo ICMbio, o cadastro nacional de unidades de conservação (CNUC), que aqui no Brasil é o órgão responsável pela gestão destas áreas, para interpretar este fenômeno. Foi feita uma análise exploratória destes dados, interpretando-os a partir das observações feitas nos trabalhos pesquisados. Pretende-se com isso ampliar as discussões sobre a temática em questão, para que ações mais concretas e efetivas sejam realizadas tendo em vista a conservação das riquezas naturais presentes no nosso planeta.

\section{Metodologia}

De acordo com Lang Silveira (1996) há sempre uma concepção epistemológica subjacente a qualquer investigação qual seja o empirismo-indutivismo (Cawthron e Rowell, 1978; Hodson, 1986; Silveira, 1989 e 1992). Para se obter sucesso em qualquer trabalho cientifico são feitas as seguintes colocações:

1 - A observação é a fonte e a função do conhecimento;

2 - O conhecimento científico é obtido dos fenômenos;

3 - A especulação, a imaginação, a intuição, a criatividade não devem desempenhar qualquer papel na obtenção do conhecimento;

4 - As teorias científicas não são criadas, inventadas ou construídas mas descobertas em conjuntos de dados empíricos (relatos de observações, tabelas laboratoriais, etc.).

Neste sentido, toda a observação e/ou experimentação estão impregnadas de pressupostos e teorias (Lang Silveira, 1996, apud Lakatos). "Observar é dirigir a atenção para algum aspecto da realidade e, portanto, a observação é antecedida por algum pressuposto ou teoria que lhe orienta. Os dados sensoriais somente adquirem significado quando interpretados". A observação e a interpretação estão indissoluvelmente ligadas. Dessa forma, o artigo em questão utilizou-se de dados retirados de agências governamentais e utilizou-se o software de código aberto $\mathrm{R}$ para fazer as análises. $\mathrm{O}$ pacote utilizado para $\mathrm{o}$ procedimento de análise foi o Tidyverse, criado por Wickham (2016).

\section{Resultados e Discussão}

\subsection{Contexto internacional}

O Parque Nacional de Yellowstone nos Estados Unidos, criado em 1872, é considerado por muitos especialistas a primeira área protegida de acordo com o quadro conceitual moderno. A criação deste parque só foi possível devido a influência de movimentos que ganharam força entre os intelectuais e artistas naquele tempo. Um desses movimentos foi o chamado 
transcendentalismo romântico, que teve como principais defensores: George Clatin (1796 - 1872) e Henry David Thoreau (1817-1862). Outro movimento que se destacava cada vez mais naquele período foi impulsionado pelo desenvolvimento das Ciências Naturais, que teve como contribuição autores como Carl Von Linné (1707 - 1778), Charles Darwin (1809 - 1882) e Alfred Russel Wallace (1823 - 1913), exercendo uma enorme influência nos círculos acadêmicos. Dessa forma, a partir destas duas visões de mundo nasceu os movimentos chamados preservacionistas e conservacionistas que influenciam as ações de conservação da natureza no século XX até hoje em vários países (Franco et al., 2015).

Com o passar do tempo, os ambientalistas em todo mundo constataram que a conservação da natureza por meio da criação de parques e áreas naturais protegidas seria uma forma eficiente de proteção ambiental e esta discussão ganhou uma dimensão internacional. Porém, com a instabilidade política existente no período do fim do século XIX até o fim da segunda guerra, avanços nessa questão foram impossibilitados. Com isso, após este período em que se obteve uma relativa paz no mundo pós-guerra, o ambiente político ocidental favoreceu para a criação de organizações internacionais dedicadas exclusivamente à proteção da natureza. Neste sentido, a recém-criada Organização das Nações Unidas para Educação, Ciência e Cultura (UNESCO) foi usada como base para a criação, em 1948, da União Internacional para a Proteção da Natureza (IUPN), que mais tarde, veio a ser denominada IUCN (International Union for Conservation of Nature) (Franco et al., 2015).

A IUCN é uma organização internacional e a maior rede de conservação ambiental no mundo. Foi fundada em Fontainebleau, França, em 1948, e sua sede está localizada em Gland, Suíça. Constitui-se de uma união democrática de membros que inclui Estados-Nação, agências governamentais, organizações não-governamentais (ONGs) e milhares de cientistas e especialistas de vários países em suas seis comissões, em uma parceria mundial. Desde sua criação, a IUCN tem focado suas ações na conservação da natureza e dos recursos naturais com especial atenção para a conservação da biodiversidade. Uma das comissões criadas pela IUCN é a Comissão Mundial de Áreas Protegidas (WCPA). Esta comissão promove um direcionamento nas ações de manejo das áreas protegidas através do estabelecimento de grupos de especialistas do mundo todo para se pensar em soluções para a gestão destas áreas, formulando diretrizes de melhores práticas (Bhandari, 2012).

Diante disso, a IUCN formula um conceito de área protegida, resultado de vários debates científicos que ampliaram o entendimento da importância das áreas protegidas. Para a IUCN (2008) uma área protegida é "um espaço geográfico claramente definido, reconhecido, dedicado e gerido, através de meios legais ou outros meios eficazes, para alcançar a conservação a longo prazo da natureza com serviços ecossistêmicos e valores culturais associados". As categorias de áreas protegidas foram definidas pela IUCN na Assembleia Geral da entidade em Buenos Aires no ano de 1994. Nesse evento, foi elaborado um sistema de categoriais que pretendeu servir como base para os sistemas nacionais e que é válido até hoje. Essa categorização divide as áreas protegidas em: Reserva Natural Estrita (Ia), Área Natural Silvestre (Ib), Parque Nacional (II), Monumento Natural (III), Área de manejo hábitats/espécies (IV), Paisagem terrestre e marinha protegida (V) e Área Protegida Manejada (VI) (Pureza, 2016).

A Figura 1 mostra o gráfico da quantidade de áreas protegidas criadas no mundo a cada ano. $\mathrm{O}$ dataset usado para a criação do gráfico, o Banco de Dados Mundial sobre Áreas Protegidas (WDPA), foi tirado do site da IUCN . O ano utilizado é o do início do estabelecimento da área como uma área protegida. Percebe-se que em um dado período desta série histórica, a quantidade de áreas criadas por ano começa a aumentar. Algumas explicações são possíveis para esse aumento, uma delas se dar pelo fato da realização das grandes Conferências realizadas neste período. A primeira delas é a Conferência das Nações Unidas sobre o Meio Ambiente Humano, realizada em Estocolmo em 1972. A segunda foi a Conferência das Nações Unidas sobre o Meio Ambiente, realizada no Rio de Janeiro no ano de 1992. Segundo Barbieri (2017), estas conferências marcam uma era de acordos multilaterais que buscam resolver os problemas ambientais, sendo as áreas protegidas um importante instrumento de conservação. 
Figura 1 - Criação de Áreas Protegidas por ano no mundo.

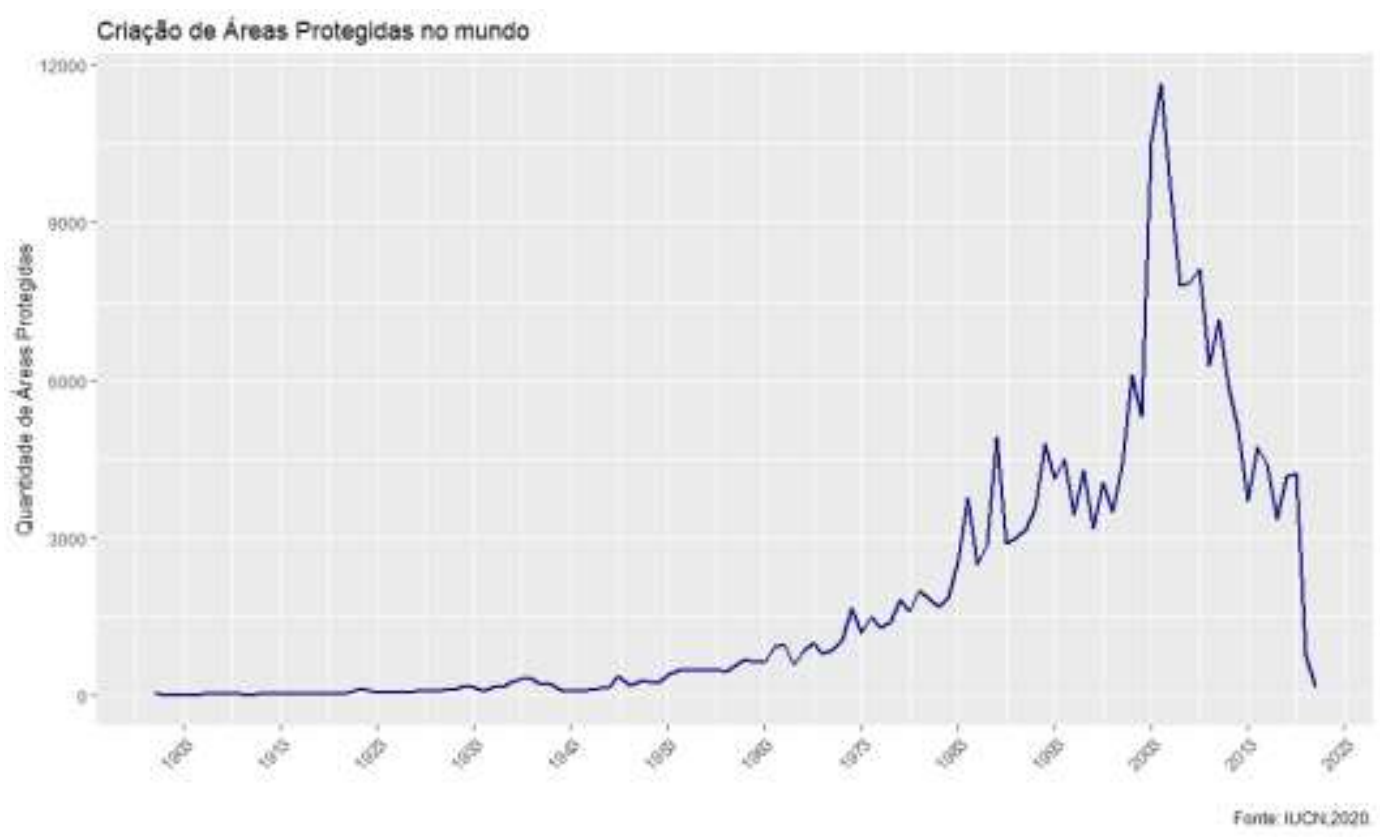

Dentro da referida Conferência das Nações Unidas sobre o Meio Ambiente, foi elaborado a Convenção da Biodiversidade (CBD), que estabeleceu algumas metas sobre a proteção da biodiversidade. Segundo Corson et al (2014), apesar de não ter se efetivado as metas propostas na CDB, que visavam a redução da degradação da biodiversidade até 2010, os países participantes da CDB foram efetivos em uma das metas que era a de manter conservado pelo menos $10 \%$ de cada bioma do mundo. Uma das causas dessa conquista se deu por causa da propagação das políticas de criação das áreas protegidas no mundo. Com isso, em uma nova reunião que aconteceu na Conferência das Partes do CDB no Japão, os países concordaram em aumentar essa meta até 2020 para obter um nível de conservação de pelo menos 17\% das áreas terrestres e das águas interiores e $10 \%$ das áreas costeiras e marinhas. Para a CDB, as áreas protegidas são consideradas o principal meio de alcançar a conservação da biodiversidade e desde da sua criação em 1992 concentra seus esforços nelas (Corson et al, 2014).

Desde então a política de criação de áreas protegidas tem sido utilizada como instrumento de conservação dos Recursos Naturais por governos em várias regiões do mundo. Todavia, a eficiência dessa política ainda tem levantado inúmeros debates entre pesquisadores e ambientalistas. Segundo Johannesen (2007), a criação de áreas protegidas foi bastante incentivada no continente africano. Entretanto, o autor observa que o cumprimento dos objetivos propostos na criação dessas áreas em muitos casos não foi efetivado. Partindo do indicador de vida selvagem presente na área, Johannsesn (2007) mostrou através de modelos econômicos-ecológicos que a expansão da área protegida pode até contribuir para a redução de estoque de animais silvestres e isso acontece frequentemente quando a produtividade do trabalho na agricultura dos habitantes locais é menor que a produtividade do trabalho na caça. Para a autora, a melhoria da produtividade agrícola das populações locais promoverá consequentemente a conservação da vida selvagem no interior das áreas protegidas.

Os autores Leberger et al. (2020) estudaram o estado de conservação e as tendências de desflorestamento global nas áreas protegidas, considerando as categorias diversas existente dentro da classificação adotada pela IUCN. Descobriram que as áreas protegidas tiveram mais perdas do que áreas desprotegidas em algumas sub-regiões, como na Europa Oriental, Mesoamérica e norte da África. Os resultados da pesquisa mostraram que em escala global as áreas protegidas tendem a apresentar resultados positivos com relação a conservação dos recursos naturais. Porém, os autores identificaram que para algumas sub-regiões o nível de desflorestamento para as categorias em que existe uma restrição maior de atuação humana é maior do que em categorias onde existe uma flexibilização. 
Em outra perspectiva, Abman (2018) analisa em que grau a corrupção, a proteção dos direitos de propriedade privada e a estabilidade política estabelecem uma relação com a pressão do desmatamento nas áreas protegidas. Usando dados espaciais explícitos sobre cobertura florestal, desmatamento, limites de áreas protegidas e características da terra, o referido autor estima os efeitos do desmatamento em áreas protegidas nos anos de 2000 a 2012 em 71 países e faz a relação com seus sistemas políticos. Para o autor, existem evidências que mostram uma correlação positiva entre o estabelecimento de um estado democrático, proteção dos direitos de propriedade e baixos níveis de corrupção com uma maior eficácia na governança das áreas protegidas.

\subsection{Contexto nacional}

Em relação ao Brasil, o termo utilizado com conceito similar para o de área protegida é unidade de conservação (UC). Sendo a primeira área protegida criada ainda no regime imperial, a Floresta da Tijuca, que depois em 1961 foi transformada em Parque Nacional da Tijuca. Porém, as unidades de conservação nos moldes atuais só foram criadas depois da Constituição de 1934 que outorgava à natureza a função de patrimônio nacional e a partir dela foram criados o Parque Nacional do Itatiaia (1937), Parque Nacional do Iguaçu (1939) e o Parque Nacional da Serra dos Órgãos (1939). Entretanto, a motivação existente até a década de 60 para a criação de unidades de conservação era de caráter estético e de acordo com circunstâncias políticas favoráveis. Ainda não havia até então, uma política centralizada de criação de áreas protegidas que tivesse como objetivo garantir a conservação de amostras representativas dos vários ecossistemas existentes no território brasileiro (Mercadante, 2001).

Antes da Constituição de 1988, que no Art. 225 impõe ao poder público a definição de espaços especialmente protegidos, existiam algumas categorias de unidades de conservação que desempenhavam objetivos distintos no ordenamento jurídico brasileiro. Porém, essas categorias ainda não eram integradas e organizadas dentro de um sistema planificado. E isso consequentemente dificultava as ações de manejo, criação e gestão dessas áreas. Além disso, existia também uma pressão de organizações internacionais como a IUCN que buscava uma padronização das áreas protegidas em todo o mundo. Tudo isso levou a uma necessidade de estabelecer os fundamentos legais de novas categorias de unidades de conservação, assim como de uma lei que organizasse todas essas categorias em um sistema ordenado que visasse o processo de seleção, criação e gestão dessas áreas com o objetivo central de conservação ambiental (Mercadante, 2001).

A Lei 9.985/00 veio para suprir essa necessidade e para efetivar os objetivos da Política Nacional de Meio Ambiente (Lei 6.938/81). A referida lei institui o Sistema Nacional de Unidades de Conservação da Natureza (SNUC) e estabelece critérios e normas para a criação, implantação e gestão das unidades de conservação. O SNUC (Sistema Nacional de Unidades de Conservação) tem como seu objetivo principal garantir a viabilidade dos objetivos nacionais de conservação da biodiversidade no longo prazo e para isso organiza em seu sistema um conjunto de áreas protegidas orientando o seu planejamento, manejo e gerenciamento. Com isso, a criação de um sistema único é considerada um marco no avanço da conservação ambiental no Brasil. O SNUC foi responsável por regularizar os critérios necessários para a criação dessas áreas, estabeleceu as categorias de manejo e definiu objetivos para os vários tipos de categorias, que anteriormente eram dispersos em variados instrumentos legais (Silva, 2015).

Segundo a SNUC (Lei 9.985/00, Art $2^{\circ}$ ) uma unidade de conservação é um "espaço territorial e seus recursos ambientais, incluindo as águas jurisdicionais, com características naturais relevantes, legalmente instituído pelo Poder Público, com objetivos de conservação e limites definidos, sob regime especial de administração, ao qual se aplicam garantias adequadas de proteção". Com relação as categorias existentes no SNUC, são divididas em dois grupos: as de Proteção Integral e as de Uso Sustentável. De acordo com a referida lei o objetivo central das Unidades de Proteção Integral é "preservar a natureza, sendo admitido apenas o uso indireto dos seus recursos naturais, com exceção dos casos previstos nesta Lei”. Já para 
a Unidade de Uso Sustentável o objetivo vai ser "compatibilizar a conservação da natureza com o uso sustentável de parcela dos seus recursos naturais”. Dentro dos grupos de Unidades de Proteção Integral e Uso Sustentável existem doze categorias. Para as de Proteção Integral as categorias são: Estação Ecológica (ESEC), Reserva Biológica (REBIO), Parque Nacional (PARNA), Monumento Natural (MONAT), Refúgio de Vida Silvestre (RVS). No caso do grupo de Uso Sustentável são: Área de Proteção Ambiental (APA), Área de Relevante Interesse Ecológico (ARIE), Floresta Nacional (FLONA), Reserva Extrativista (RESEX), Reserva de Fauna (RFAU), Reserva de Desenvolvimento Sustentável (RDS) e Reserva Particular do Patrimônio Natural (RPPN).

A Figura 2 mostra a quantidade de UCs criadas por ano no Brasil. Percebe-se que nos anos 70 houve um aumento significativo na criação destas áreas seguindo a tendência do resto do mundo, como mostrado na Figura 1. Nos anos posteriores a criação do SNUC, observa-se que o número de UCs criadas cresceu e depois manteve-se mais ou menos constante com leves variações em alguns anos. Entretanto, alguns problemas envolvendo sua expansão e gestão dos territórios criados foram observados desde então. Segundo Santos (2018), por mais que muitas UCs tenham sido criadas desde o SNUC poucas tem alcançado efetividade em suas finalidades propostas. O autor estudou as UCs na região do Cerrado, e identificou que houve de fato muitos espaços protegidos criados nos últimos anos, mas segundo o autor a gestão territorial dessas áreas ainda precisa melhorar. Para Santos (2018), a maioria das UCs criadas nessa região se encontram em locais impróprios para a utilização econômica dos recursos, com solos não aproveitáveis para atividades agrícolas. Isto se dá segundo ele, devido ao fato de que as áreas com os solos mais aproveitáveis já estarem sendo usadas por atividades agrícolas de grande extensão sem levar em conta seus prováveis potenciais ecológicos, ficando as UCs com áreas frequentemente distantes dos polos de atração e com pouca biodiversidade. $\mathrm{O}$ autor defende que as localizações das UCs têm que ser em locais estratégicos do ponto de vista ecológico, para que se ocorra uma conservação efetiva.

Figura 2 - Criação de Unidades de Conservação por ano no Brasil.

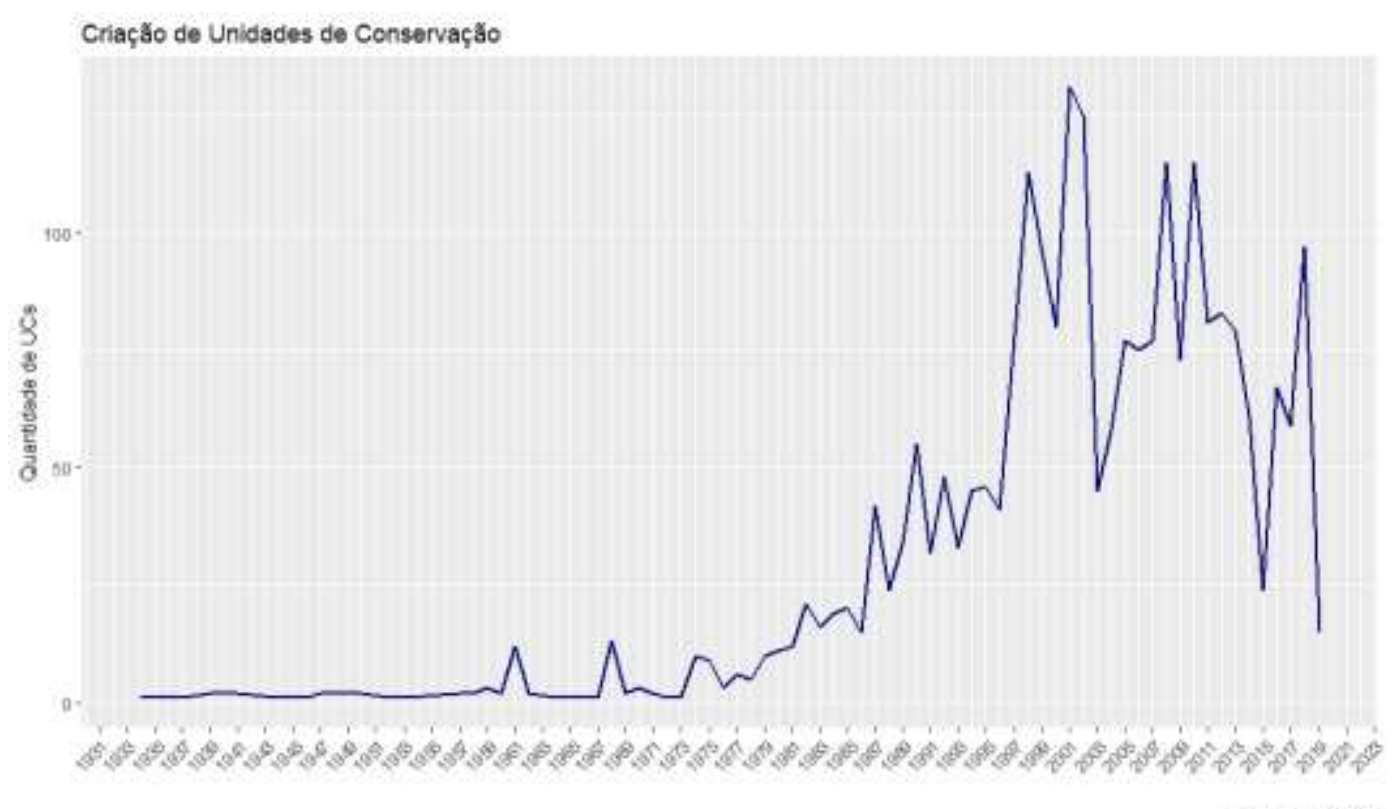

A Figura 3 é um gráfico de barras que mostra a proporção da quantidade de UCs por categoria em cada bioma brasileiro. As diferentes cores indicam os sete biomas presentes no Brasil. Por exemplo, a cor azul representa as unidades de conservação pertencentes ao bioma Mata Attântica. Para a categoria APA, a quantidade de UCs criadas no bioma Mata Atlântica representa mais de $50 \%$ das UCs criadas para esta categoria específica. A partir da observação do gráfico podemos 
concluir que para a categoria APA, o bioma Mata Atlântica é o mais frequente dentre todas as UCs criadas no bioma Mata Atlântica, sendo a categoria APA a mais adotada para instituir UCs neste bioma. Seguindo esse raciocínio observamos que para as categorias FLONA, RDS e RESEX o bioma Amazônia representa a maior proporção. O bioma da Caatinga representado pela cor dourado aparece com maior frequência na categoria de Monumento Natural, assim como o bioma Cerrado.

Figura 3 - Categorias de Unidades de Conservação por Bioma no Brasil.

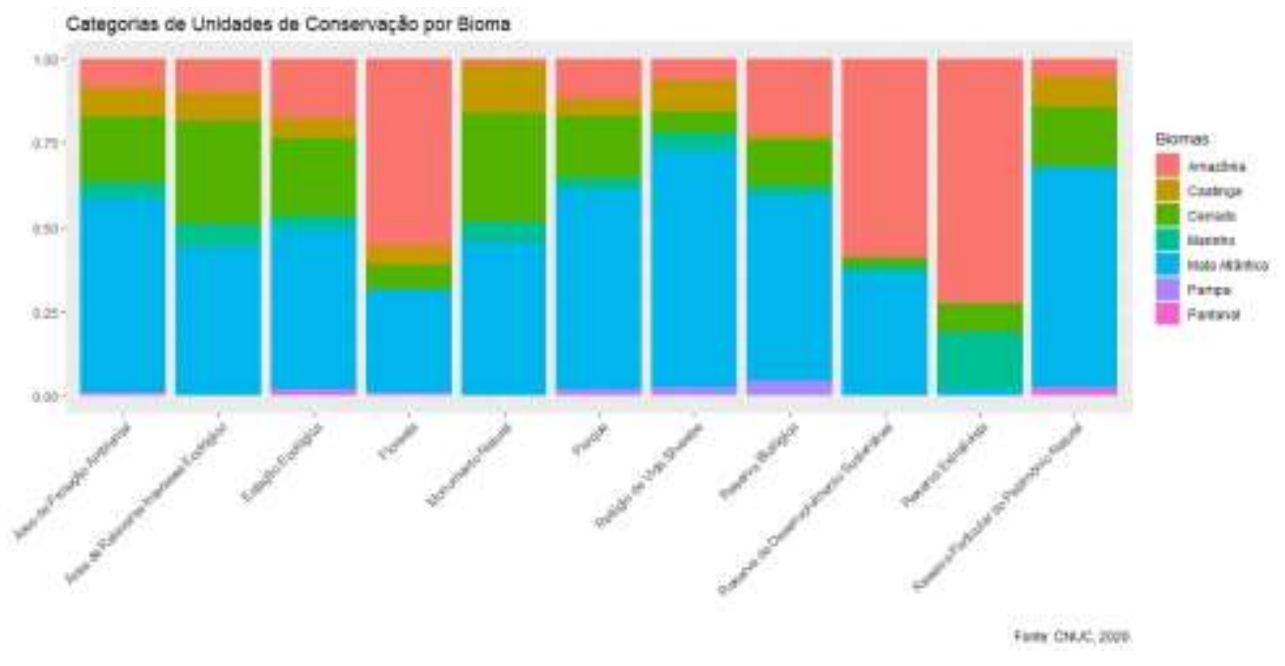

Tofeti (2018) estudou as transformações ocorridas no uso do território promovidas pelas UCs. Para o autor não é possível predizer que as Unidades de Proteção Integral promovem maiores mudanças em seu território que as de Uso Sustentável. O autor observa que para a real implementação da SNUC no território se faz necessário uma atitude de complementariedade com os diversos atores conflitantes envolvidos na região da UC. Ele considera necessário alinhar os diversos fatores envolvidos em uma UC, tanto de natureza ambiental quanto social ou econômica, para que a gestão territorial tenha mais "sucesso". Segundo ele, a gestão das UCs quando ocorre com uma visão mais preservacionista pode levar a uma falta de participação da população local e dos atores envolvidos na região no qual afetaria a efetividade da política ambiental.

Em outra perspectiva, Freire et al. (2020) avaliou as UCs de proteção integral no bioma Caatinga usando o método de mapeamento e análise espectro-temporal com o objetivo de averiguar o estado atual de conservação da vegetação nessas áreas. Os pesquisadores observaram que que 18,68\% de área das UCs no bioma Caatinga foram degradadas no período entre $2000 \mathrm{e}$ 2017 e isto representa uma perda de biodiversidade considerável para o bioma, tendo em vista que historicamente esse ecossistema já tem sido negligenciado pelas políticas públicas. Uma das possíveis causas para essas perdas segundo o autor é o baixo nível de indicadores socioeconômicos municipais como o de educação e a renda e isso leva a uma falta de participação da população local nas decisões da gestão territorial.

\section{Conclusão}

$\mathrm{O}$ artigo em questão contribuiu para o aprofundamento desta temática, tendo em vista a melhoria do quadro atual na proteção ambiental. Foi apresentado uma revisão bibliográfica dos principais trabalhos publicados sobre o tema das áreas protegidas tanto em um contexto internacional quanto em um contexto nacional. A análise exploratória dos dados de áreas protegidas pelo mundo foi feita e interpretada a partir das observações feitas nos trabalhos pesquisados.

Percebe-se que a partir da década de 60/70 a quantidade de áreas criadas por ano começa a aumentar. Algumas 
explicações são possíveis para esse aumento, uma delas se dar pelo fato da realização das grandes Conferências realizadas neste período. A primeira delas é a Conferência das Nações Unidas sobre o Meio Ambiente Humano, realizada em Estocolmo em 1972. A segunda foi a Conferência das Nações Unidas sobre o Meio Ambiente, realizada no Rio de Janeiro no ano de 1992. No Brasil este mesmo movimento também aconteceu. Principalmente depois da instituição da SNUC no ano de 2000, como mostrado na Figura 2.

Através das referências trazidas no trabalho, observa-se que mesmo aumentando o número de áreas criadas com a finalidade de proteção ambiental em todo o globo, a conservação dos ecossistemas ainda carece de bastante atenção dos governantes em várias partes. Muito devido ás fragilidades institucionais existentes em vários países considerados em desenvolvimento, muitos biomas destas regiões ainda se encontram em perigo de serem gradualmente devastados, levando assim a degradação de muitas riquezas naturais que poderiam servir ao bem comum da sociedade. Aqui no Brasil, este problema tem sido colocado em evidência, principalmente pela fragilidade existente no sistema democrático nacional que ainda carece de adesão entre a população.

\section{Referências}

Aguiar, C. C. D. (2013). Externalidades positivas e custos dos espaços protegidos em áreas de produção sucroenergética (Doctoral dissertation, Universidade De São Paulo).

Andam, K. S., Ferraro, P. J., Pfaff, A., Sanchez-Azofeifa, G. A., \& Robalino, J. A. (2008). Measuring the effectiveness of protected area networks in reducing deforestation. Proceedings of the national academy of sciences, 105(42), 16089-16094.

Barbieri, J. C. (2017). Gestão ambiental empresarial. Saraiva Educação SA.

Bhandari, M. (2012). International Union for Conservation of Nature. The Wiley-Blackwell Encyclopedia of Globalization.

Borrini-Feyerabend, G. R. A. Z. I. A., Dudley, N., Jaeger, T., Lassen, B., Broome, N. P., Phillips, A., \& Sandwith, T. (2017). Governança de Áreas Protegidas: da compreensão à ação. Série Diretrizes para melhores Práticas para Áreas Protegidas, (20).

Brockington, D., \& Igoe, J. (2006). Eviction for conservation: a global overview. Conservation and society, 424-470.

Cawthron, E. R., \& Rowell, J. A. (1978). Epistemology and Science Education. Epistemology and Science Education, 5, $31-59$.

Chang, W. (2018). R graphics cookbook: practical recipes for visualizing data. O'Reilly Media.

Dixon, J. A., \& Sherman, P. B. (1991). Economics of protected areas. Ambio, 68-74.

Ervin, J. (2003). Protected area assessments in perspective. BioScience, 53(9), 819-822.

Gamarra, N. C., Correia, R. A., Bragagnolo, C., Campos-Silva, J. V., Jepson, P. R., Ladle, R. J., \& Malhado, A. C. M. (2019). Are protected areas undervalued? An asset-based analysis of Brazilian protected area management plans. Journal of environmental management, $249,109347$.

Hannah, L., Midgley, G., Andelman, S., Araújo, M., Hughes, G., Martinez-Meyer, E., \& Williams, P. (2007). Protected area needs in a changing climate. Frontiers in Ecology and the Environment, 5(3), 131-138.

Hodson, Derek (1986). Philosophy of Science and Science Education. Philosophy of Education. 20 (2):215-225.

Scherl, L. M., Wilson, A., Wild, R., Blockhus, J., Franks, P., McNeely, J. A., \& McShane, T. O. (2006). As áreas protegidas podem contribuir para a redução da pobreza. Oportunidades e limitações. Reino Unido: IUCN, 60.

Silveira, F. L. D. (1989). A filosofia de Karl Popper e suas implicações no ensino da ciência. Caderno catarinense de ensino de física. Florianópolis. Vol. 6, n. 2 (ago. 1989), p. 148-162.

Silveira, F. L. D. (1992). Uma epistemologia racional-realista e o ensino da Física.

Silveira, F. L. D. (1996). A metodologia dos programas de pesquisa: a epistemologia de Imre Lakatos. Caderno catarinense de ensino de fisica. Florianópolis. Vol. 13, n. 3 (dez. 1996), p. 219-230.

Vallejo, L. R. (2002). Unidade de conservação: uma discussão teórica à luz dos conceitos de território e políticas públicas. Geographia, 4(8), 57-78.

Walde, J., Huy, D., Tappeiner, U., \& Tappeiner, G. (2019). A protected area between subsistence and development. International Journal of the Commons, $13(1)$.

Wardropper, C. B., Mase, A. S., Qiu, J., Kohl, P., Booth, E. G., \& Rissman, A. R. (2020). Ecological worldview, agricultural or natural resource-based activities, and geography affect perceived importance of ecosystem services. Landscape and Urban Planning, 197, 103768.

Wickham, H., \& Grolemund, G. (2016). R for data science: import, tidy, transform, visualize, and model data. O'Reilly Media, Inc. 